Meta

Journal des tradlucteurs

Translators' Journal

\title{
Lexique anglais-français du microfilm
}

\section{Ray J. Pollet}

Volume 19, numéro 3, septembre 1974

URI : https://id.erudit.org/iderudit/003852ar

DOI : https://doi.org/10.7202/003852ar

Aller au sommaire du numéro

Éditeur(s)

Les Presses de l'Université de Montréal

ISSN

0026-0452 (imprimé)

1492-1421 (numérique)

Découvrir la revue

Citer cet article

Pollet, R. J. (1974). Lexique anglais-français du microfilm. Meta, 19(3), 158-164. https://doi.org/10.7202/003852ar d'utilisation que vous pouvez consulter en ligne.

https://apropos.erudit.org/fr/usagers/politique-dutilisation/ 


\title{
LEXIQUE ANGLAIS-FRANÇAIS DU MICROFILM
}

Ce lexique ne constitue qu'un travail de base, qu'il faudra compléter et étoffer à mesure que les découvertes technologiques viendront développer toujours davantage le monde fascinant du microfilm.

La plupart des termes assemblés ici et traduits par l'auteur proviennent de brochures Kodak et de documents techniques de référence.

alphanumerical
aluminized
aluminized plastic wrapping
automated
automated retrieval
automatic
automatic feeder
automatic scanning
automatic stacker

\author{
alphanumérique \\ aluminisé \\ emballage de plastique aluminisé, \\ présentation en plastique aluminisé \\ automatisé \\ repêrage automatisé, recherche automatisée \\ automatique \\ dispositif d'alimentation automatique, \\ distributeur automatique \\ exploration automatique, repérage \\ automatique, recherche automatique \\ dispositif d'empilement automatique, \\ empileur automatique
}




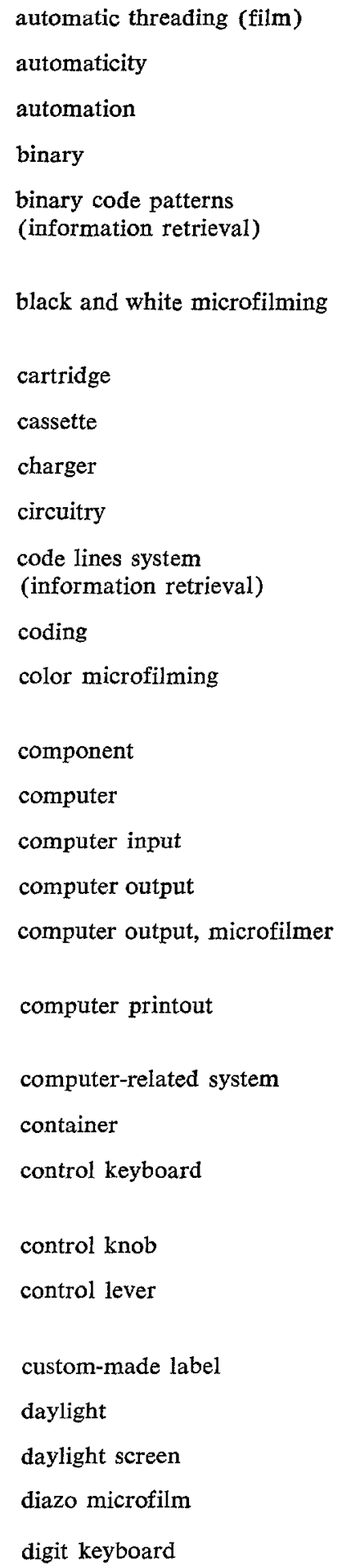




digit selector
document jogging
dry ink
duo method
duplex method
duplication
elevation control
endorser
extraneous light
feeder
feeder /stacker combination
image control method
filing
film
film strip
film transport
filming unit
flash card
(retrieval)
front projection optics
gamma
graduated scale (retrieval)
identionsing (camera)
identification
identification number
idication symbol
image control keyboard
imaliol
imase

sélecteur numérique

taquage des documents

encre sèche

procédé duo, microfilmage en duo

procédé duplex, microfilmage en duplex

reproduction, duplication

réglage en hauteur

endorseur, dispositif d'endorsement

lumière parasite, luminosité parasite, éclairage superflu

dispositif d'alimentation, plateau d'alimentation, distributeur

dispositif combiné d'alimentation et d'empilement, distributeur-empileur

classement

film, pellicule

bande microfilmée

translation du film, entraînement du film appareil de filmage, caméra (microfilm)

fiche signalétique, fiche de repérage, repère "flash" (localisation)

système optique à projection frontale

gamma, facteur de contraste

échelle graduée (localisation)

boîtier, carter (caméra)

indentification

numêro d'identification

symbole d'identification, sigle d'identification

image, cliché, vue

sélection des images, repérage des images

clavier de présélection des images, clavier de recherche des images

procédé de repérage des images, système de recherche des images

sélecteur numérique des clichés microfilmés

localisation des images, repérage des clichés microfilmés 


\author{
image rotation \\ index system \\ index tab \\ indexing \\ indexing scale \\ information \\ information retrieval
}

interchangeable lens system

jacket

jogging

keyboard

label

labelling

location (image)

magazine

magnetic tape

magnification ratio

management of information

manual

manual feeder/stacker

manual retrieval (information)

microfiche

microfiche edition

microfile

microfilm

microfilm copy

microfilm system

microfilmer (camera)

microfilmer (operator) rotation des images

système d'indexage, procédé d'indexage

onglet, repère d'indexage

indexage

grille d'indexage

information, données

recherche de l'information,

repérage de l'information,

localisation de l'information

objectifs interchangeables

jacket, jacquette

taquage

clavier, tabulateur

étiquette

étiquetage

localisation, repérage, recherche (images)

magasin, chargeur

bande magnétique, ruban magnétique

rapport d'agrandissement,

taux d'agrandissement, rapport d'amplification

gestion de l'information, traitement de l'information

manuel

distributeur-empileur manuel

repérage manuel, recherche manuelle (information)

microfiche

microversion

microdossier

microfilm

microcopie

système de microfilmage, appareil de microcopiage

appareil de microfilmage,

caméra de microfilmage, microfilmeur, microcopieur (appareil de prise de vues)

microfilmeur, microcinéaste (opérateur) 


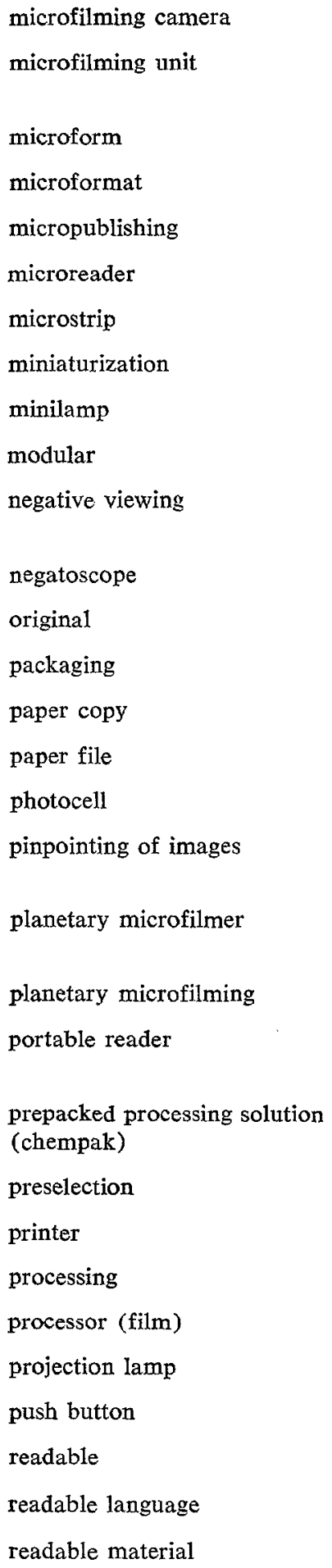

caméra de microfilmage

appareil de microfilmage, système complet de microfilmage

microformule

microformat

micropublication, microédition

microlecteur

bande microfilmée

miniaturisation, réduction

mini-lampe

modulaire

examen par transparence, projection par transparence

négatoscope

original

présentation, emballage

copie papier

dossier papier

cellule photoélectrique

repérage des images, identification des clichés

appareil de microfilmage statique, microfilmeur statique

microfilmage statique

appareil de lecture portatif, lecteur de faible encombrement

ensemble chimique, conditionnement chimique (chempak)

présélection

imprimante

traitement, développement

appareil de traitement (film)

lampe de projection

poussoir, bouton-poussoir

lisible, compréhensible

langage lisible

données lisibles 


\author{
reader \\ reading screen \\ reading system \\ readout panel \\ record (to) \\ records \\ reduction ratio \\ reel \\ reflective substance \\ retrieval \\ retrieve (to) \\ roll of film \\ rotary microfilmer
}

rotary microfilming

scanning

screen (to)

screen

screening

search button

searching

selection

selectivity

self-threading

sequential numbering (retrieval)

silver microfilm

sophisticated circuitry

sophistication

spent solution

stacker

storage

stored information appareil de lecture, lecteur

écran de lecture

système de lecture, procédé de lecture

fenêtre indicatrice

enregistrer, noter

enregistrements, documents, dossiers, données, archives

rapport de réduction, taux de réduction

bobine

substance à pouvoir réfléchissant

repérage, recherche, localisation

repérer, localiser

rouleau de film

appareil de microfilmage dynamique, microfilmeur dynamique, microfilmeur en continu

microfilmage dynamique, microfilmage en continu

exploration, analyse, examen

visionner, étudier

écran

visionnement, lecture

bouton de repérage, bouton de sélection repérage, recherche, localisation

sélection, choix, recherche

sêlectivité

auto-amorçage, amorçage automatique

comptage séquenitel repérage séquentiel (localisation)

microfilm à support argentique

montage complexe, montage poussé, montage raffiné

raffinement, complexité

solution usée

dispositif d'empilement, empileur

storage, mémoire

information mémorisée, données mémorisées 
strip holder

tab card

toner

transfer speed

translation speed

ultrafiche

vesicular microfilm

viewing

viewing angle

voltage stabilization

wrapping support pour bandes microfilmées

carte d'indexage, fiche de repérage, carte-index

produit de virage, vireur, révélateur, modificateur de contraste, changeur de tonalité

vitesse de transfert, cadence de transfert

vitesse de transposition,

cadence de transposition

ultrafiche

microfilm à support vésiculeux

visionnement, lecture

angle de visionnement

stabilisation de tension

emballage, présentation

Ray. J. Pollet 